ORIGINAL ARTICLE

\title{
Leftward Optical Shift Induces Bias in Line Bisection: A Study with Healthy Subjects Using a Head-mounted Display
}

\author{
Taku Numao, ${ }^{\text {a, e }}$ Yuri Fujita, ${ }^{\mathrm{b}}$ Kyohei Ichikawa, ${ }^{\mathrm{c}}$ Shogo Ide, ${ }^{\mathrm{c}}$ Hidekazu Katori, ${ }^{\mathrm{c}}$ Tomoko Shimada, ${ }^{\mathrm{d}}$ \\ Kohei Ota, ${ }^{d}$ Yumi Ikeda, PhD ${ }^{\mathrm{e}}$ and Kazu Amimoto, PhD ${ }^{\mathrm{e}}$
}

\begin{abstract}
Objective: The purpose of this study was to verify the effect on spatial perception in healthy young subjects of an unconscious leftward optical shift created by a head-mounted display (HMD) with an offset camera. Methods: We recruited 40 healthy right-handed adults who were divided into four groups according to the hand used in the tests and the visual direction displayed by the HMD (centered or $10^{\circ}$ left). Each of the four groups $(n=10)$ undertook line bisection tasks across four combinations of variables: using a finger/stick or a mouse to point at a touch panel located 60 or $120 \mathrm{~cm}$ away from the subject. Results: According to the results, regardless of the hand used, when the index finger or a stick was used (reaching condition), the line bisection point was displaced significantly to the left of the center. Additionally, a major left-displacement trend was observed in the short-distance reaching task, which did not require the use of a stick. In contrast, the long-distance task required a stick to be used, and the left displacements were all smaller than those for the short-distance tasks that used the index finger. Conclusion: This finding may be explained by the subjects having sufficient experience coordinating hand and eye movements in the condition where they used their dominant hand and reached with their own arms without using a stick.
\end{abstract}

Key Words: head-mounted display; line bisection bias; unilateral spatial neglect

\section{INTRODUCTION}

Cerebrovascular disease leads to problems with balance and spatial perception, which can cause many difficulties in daily life. In particular, hemiplegia accompanied by left-sided unilateral spatial neglect (USN), which occurs as a result of damage to the right hemisphere, often leads to walking difficulties and other symptoms that extend hospital stays $^{1)}$ and limit personal pastimes and activities of daily living. ${ }^{2)}$ Consequently, the prognosis for USN patients is often considered to be poor. USN is a syndrome defined as "a consistent, exaggerated spatial asymmetry in processing information in bodily and/or extrabodily space due to an acquired cerebral lesion," ${ }^{3)}$ and is a condition that leads to spatial attention bias toward the contralateral side. As a result, left-sided USN patients continually look to their right, but are often not conscious of doing so. Moreover, this leads to a vicious cycle that makes it difficult for a patient to direct his or her attention to the left. When first breaking this vicious cycle during treatment, patients are commonly directed to turn their eyes to the left. However, there is no clear understanding of how the state of looking at the right affects USN patients' spatial perception.

Prism glasses, which constitute a tool for shifting vision, remain fixed at a specific angle throughout their use. However, in place of prism glasses, a head-mounted display

Received: November 6, 2018, Accepted: February 11, 2019, Published online: February 26, 2019

a Japanese School of Technology for Social Medicine, Koganei, Tokyo, Japan

b St. Marianna University School of Medicine, Yokohama City Seibu Hospital, Yokohama, Kanagawa, Japan

c Niiza Hospital, Niiza, Saitama, Japan

d Higashiyamato Hospital, Higashiyamato, Tokyo, Japan

e Department of Physical Therapy, Graduate School of Human Health Sciences, Tokyo Metropolitan University, Tokyo, Japan Correspondence: Kazu Amimoto, PhD, PT, Tokyo Metropolitan University, 7-2-10 Higashi-Ogu, Arakawa-ku, Tokyo 116-8551, Japan,

Email: amimoto@tmu.ac.jp

Copyright (C) 2019 The Japanese Association of Rehabilitation Medicine 
(HMD) with a movable camera installed on the front can be used to project the camera image onto the HMD. In this way, the visual direction can be more freely adjusted, i.e., the HMD and camera can be used to easily deviate the landscape optically in a manner analogous to using prisms of different power. Using this approach, we assessed the spatial perception of healthy subjects in conditions where the landscape was relatively shifted to the left, as would occur in patients with left-sided USN.

To replicate the prior spatial perception research conducted by Longo and Lourenco, ${ }^{4}$ the present study used line bisection tasks. It was recently shown that the line bisection test is not sufficient to diagnose USN. ${ }^{5,6)}$ However, among the several USN tests, we considered that it would best reflect the effect of a simple optical shift, and therefore we adopted the line bisection test in this study. Longo and Lourenco assessed spatial perception via the use of sticks and lasers in line bisection tests in healthy subjects with no optical shift. They found that regardless of the changes in perspective, line bisection was displaced slightly to the left when sticks or lasers were used. The tests conducted by Longo and Lourenco involved the use of the right hand only; however, in the present study, we added a comparison between the left and right hands. This was done to assess the bias resulting from the direction of the torso's rotation and the direction of balance movements to accommodate the use of the opposite hand during the reaching task. The purpose of this study was to verify the effect of an unconscious optical shift to the left in spatial perception in healthy young subjects.

\section{METHODS}

\section{Subjects}

We recruited 40 healthy right-handed adults (average age: $22.0 \pm 1.2$ years; 28 women and $12 \mathrm{men}$ ). The subjects were divided into four groups: (1) those using the right arm with a left optical shift, (2) those using the right arm with a centered optical shift, (3) those using the left arm with a left optical shift, and (4) those using the left arm with a centered optical shift. An HMD (Oculus VR, LLC; Oculus Rift) with an attached webcam was fitted to the subjects' heads. The camera angle was centered such that the visual direction was also centered and tilted horizontally to the right by $10^{\circ}$ (the yaw angle) for the $10^{\circ}$ left optical shift group (Fig. 1).

Written informed consent was obtained from all participants after a full explanation of the experimental procedure was provided, and the study design was approved by the appropriate ethics review board.

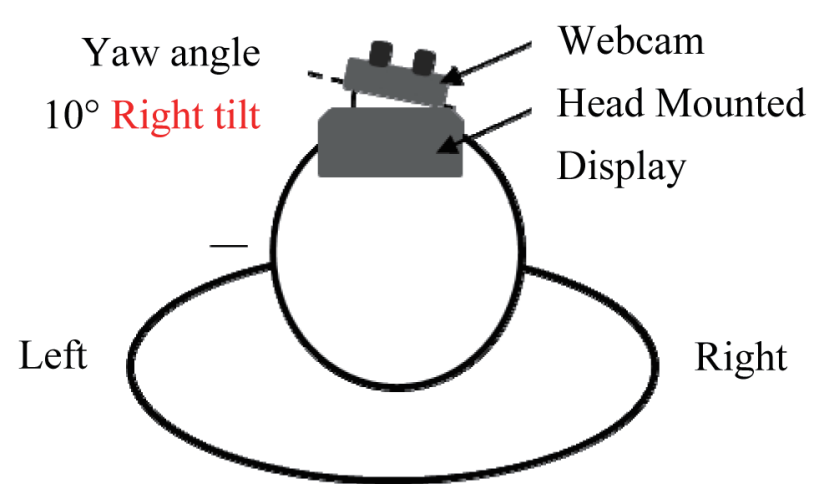

Fig. 1. Head-mounted display equipped with a webcam set up to provide a view optically shifted to the left.

\section{Line Bisection Test}

Each of the four groups underwent testing across four combinations of variables, implemented at random. The subjects used a finger/stick or mouse to point at a touch panel located 60 or $120 \mathrm{~cm}$ away from the subject (Table 1).

The subjects were seated such that they could not hold their heads in a fixed position. A $12-\mathrm{cm}$ high and $15-\mathrm{cm}$ wide piece of black cardboard was mounted underneath the webcam so that the limb or stick could not be seen during the test until just before the line segment was presented.

A 32-inch liquid-crystal display monitor (Iiyama Corporation; ProLite X3291HS) fitted with a touch panel (Xiroku Inc.; XT-AIX3291HS-B1) was installed 60 or $120 \mathrm{~cm}$ away from the subject's sternum. Using their index finger or a mouse pointer for the short distance $(60 \mathrm{~cm})$ and a $75-\mathrm{cm}-$ long stick or a mouse pointer for the long distance $(120 \mathrm{~cm})$, the subjects were asked to locate the center of the presented line segment. This position along the $\mathrm{x}$-axis, indicated by the subject, was recorded for each of nine trials. To ensure that the straight line remained equally visible regardless of the subject's distance from the monitor, a 1-mm-thick line 15 , 20 , or $25 \mathrm{~cm}$ in length was used for the $60-\mathrm{cm}$ distance, and a 2 -mm-thick line 30,40 , or $50 \mathrm{~cm}$ in length was used for the $120-\mathrm{cm}$ distance. Each item appeared nine times, with lengths of 15, 20, and $25 \mathrm{~cm}$ appearing at random.

\section{Analysis}

The measured value of the line bisection was calculated for each presented item to determine the bias ratio from the center point using the following formula: bias from center point on the $\mathrm{X}$ axis $(\mathrm{mm}) * 100 /$ half line length $(\mathrm{mm})$. The average value across the nine measurements and a standard deviation for each was calculated. Note that the calculated values set 


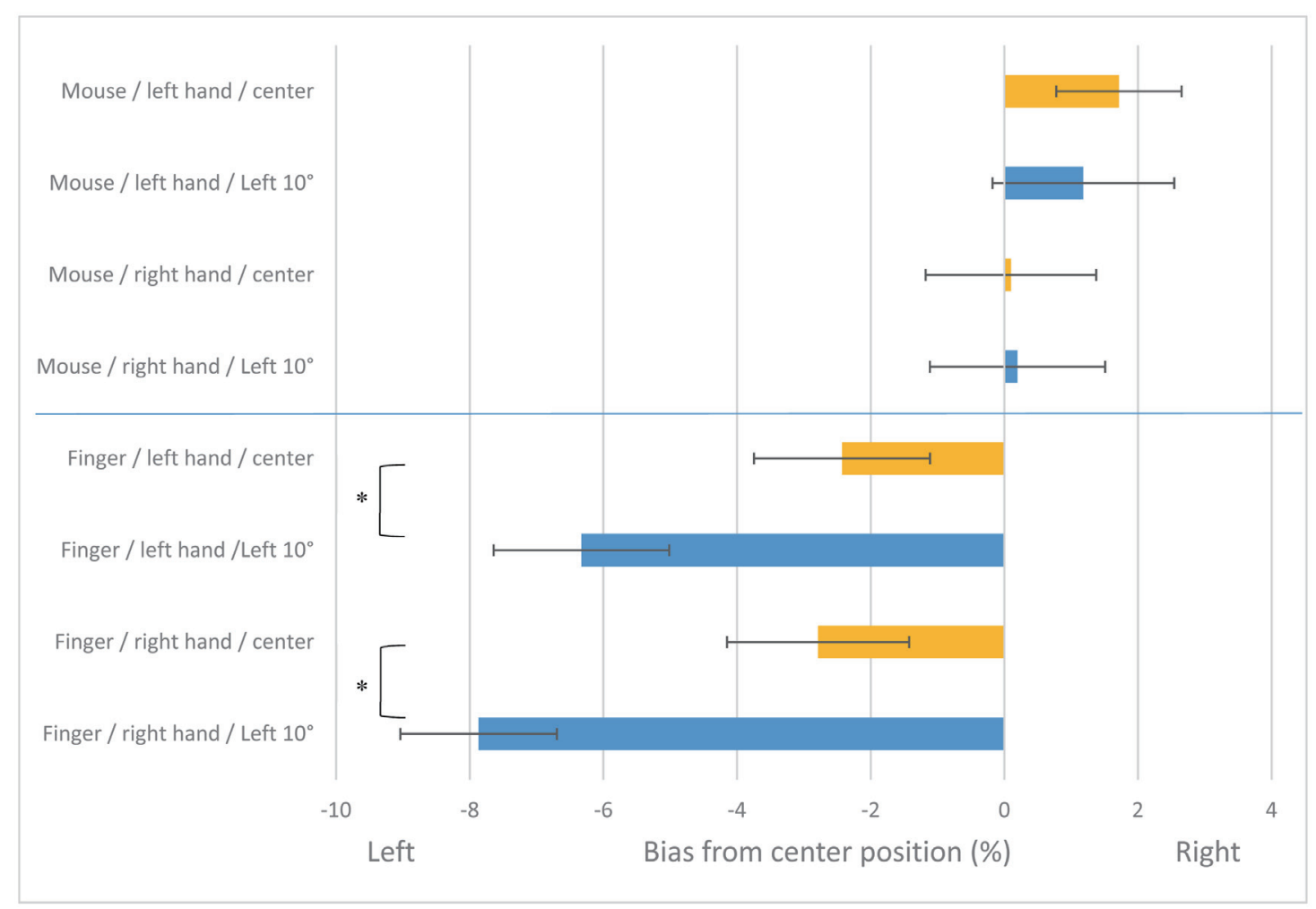

Fig. 2. Judgment of the center point of various lines at a distance of $60 \mathrm{~cm}$ from the touch panel (bars represent the standard error). $* \mathrm{P}<0.05$.

the center point at zero; therefore, bias to the right of the center point was recorded as a positive value and bias to the left of the center point was recorded as a negative value.

An independent samples $t$-test was performed to compare the results from the centered vision with those from the vision optically shifted to the left for each item in each group. Statistical software (IBM Corp.; SPSS v. 23) was used, and the level of significance was set at $\mathrm{P}<0.05$.

\section{RESULTS}

For a centered optical shift, for line segments shown on a touch panel located $60 \mathrm{~cm}$ from the subject, and bisection indicated using the right index finger, the bisection point was found to be located at an average position $2.8 \pm 1.4 \%$ to the left of the correct position. When the optical shift was deviated $10^{\circ}$ to the left, the bisection point was located at a position $7.9 \pm 1.2 \%$ to the left. When the same test was performed using the left hand and the centered optical shift, the line bisection point was $2.4 \pm 1.3 \%$ to the left, and when the optical shift was deviated $10^{\circ}$ to the left, the bisection point was $6.3 \pm 1.3 \%$ to the left (Fig. 2). When the monitor was located $120 \mathrm{~cm}$ from the subject and a stick was used with the right hand for the bisection test, for the centered optical shift, the line bisection point was $2.6 \pm 0.8 \%$ to the left. When the optical shift was deviated $10^{\circ}$ to the left, the bisection point was $3.9 \pm 0.8 \%$ to the left (Fig. 3). In all line bisection tasks in which the index finger or stick was used, a significant difference $(\mathrm{P}<0.05)$ was observed between the centered optical shift's bisection points and the bisection points deviated to the left. No significant differences were observed when the mouse pointer was used (Table 2).

To summarize, regardless of the hand used, when the index finger or stick was used (hereinafter, this condition is called the reaching condition), the line bisection point was displaced significantly to the left of the correct position. Moreover, during the reaching condition, the centered optical shift group had an average displaced line bisection point of $2.1 \pm 1 \%$. Jewell and McCourt ${ }^{7)}$ previously referred to this type of displacement as "pseudoneglect." Additionally, a major left-displaced trend was observed in the short distance task in which tools were not used, compared with a less pronounced left-displaced trend in the long-distance task in which a stick was used. 


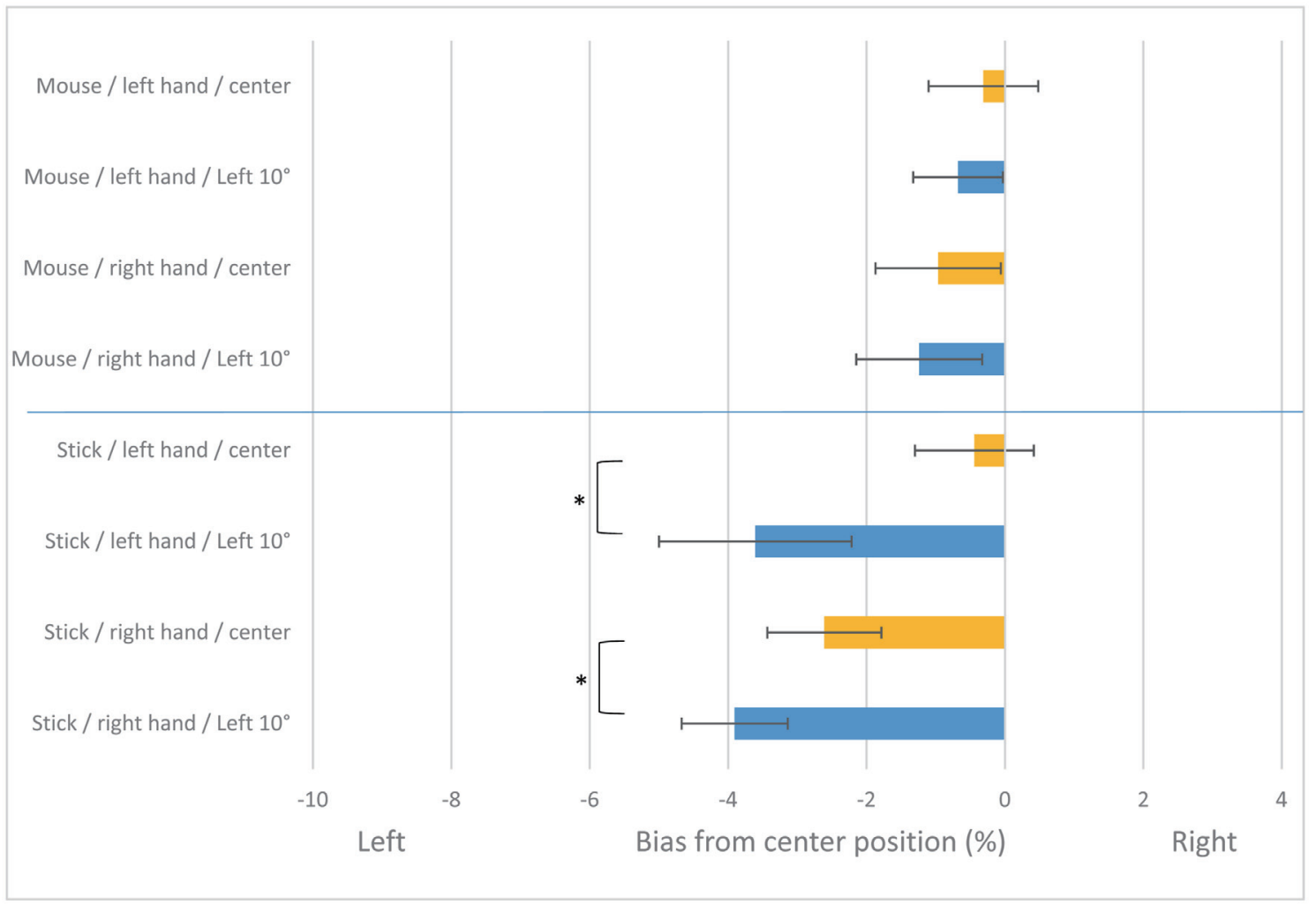

Fig. 3. Judgment of the center point of various lines at a distance of $120 \mathrm{~cm}$ from the touch panel (bars represent the standard error). $* \mathrm{P}<0.05$.

Table 1. Experimental groups and conditions

\begin{tabular}{cccc}
\hline \multicolumn{2}{c}{ Group } & \multicolumn{2}{c}{ Condition } \\
\hline Hand used & Optical shift & Device used & Distance $(\mathrm{cm})$ \\
Right & Center & Index finger (stick)* & 60 \\
Left & $10^{\circ}$ left shift & Mouse & 120 \\
\hline
\end{tabular}

*For the near distance $(60 \mathrm{~cm})$, the index finger was used; for the far distance $(120 \mathrm{~cm})$, a stick was used.

\section{DISCUSSION}

In this study, we observed the effect of an unconscious left optical shift in spatial perception in healthy young subjects. When reaching actions were performed, the results showed a leftward bias in the line bisection point independent of the distance from the screen and the hand used. This finding is the opposite of that observed in real USN cases. The performance of reaching actions requires hand-eye coordination and arm proprioception based on visual cues. In this study, visual information was displaced to the left in the HMD relative to the position of the real object (i.e., the line segment) because the camera was tilted to the right. Subjects were instructed to point to the center of the real line (Fig. 4). At the start of this reaching task, the stick or arm was outside the field of view. As a result, the subjects tended to reach out with their arm or the stick at a leftward angle. At the end of the reaching action, when subjects could observe their arm or stick on the display, they noticed that the arm or stick was off-centered and biased to the left. The subjects then attempted to amend this based on the visual information received via the display, but because of the retention of dominant visual information that had just been presented displaying the line segment to the left of where it was in actuality, participants moved their gesture only slightly to the left.

Conversely, the group that used the mouse pointer was in- 
Table 2. Judgment of the midpoint position of the line segment by each group

\begin{tabular}{|c|c|c|c|c|c|c|c|c|c|}
\hline \multirow[t]{2}{*}{ Tool used } & \multirow{2}{*}{$\begin{array}{l}\text { Hand } \\
\text { used }\end{array}$} & \multirow{2}{*}{$\begin{array}{l}\text { Optical } \\
\text { shift }\end{array}$} & \multicolumn{3}{|c|}{$60 \mathrm{~cm}$} & \multicolumn{4}{|c|}{$120 \mathrm{~cm}$} \\
\hline & & & Average $(\%)$ & \pm & SE $(\%)$ & & Average (\%) & \pm & SE $(\%)$ \\
\hline \multirow[t]{4}{*}{ Mouse } & \multirow[t]{2}{*}{ Left } & Center & 1.7 & \pm & 0.9 & & -0.3 & \pm & 0.8 \\
\hline & & Left & 1.2 & \pm & 1.4 & & -0.7 & \pm & 0.6 \\
\hline & \multirow[t]{2}{*}{ Right } & Center & 0.1 & \pm & 1.3 & & -1.0 & \pm & 0.9 \\
\hline & & Left & 0.2 & \pm & 1.3 & & -1.2 & \pm & 0.9 \\
\hline \multirow{4}{*}{$\begin{array}{l}\text { Index finger/ } \\
\text { stick }\end{array}$} & \multirow[t]{2}{*}{ Left } & Center & -2.4 & \pm & 1.3 & & -0.4 & \pm & 0.9 \\
\hline & & Left & -6.3 & \pm & 1.3 & & -3.6 & \pm & 1.4 \\
\hline & \multirow[t]{2}{*}{ Right } & Center & -2.8 & \pm & 1.4 & & -2.6 & \pm & 0.8 \\
\hline & & Left & -7.9 & \pm & 1.2 & & -3.9 & \pm & 0.8 \\
\hline
\end{tabular}

Bias to the right of the midpoint is depicted as a positive number and bias to the left is depicted as a negative number (as a percentage).

$* \mathrm{P}<0.05$.

SE, standard error.

VISUAL DIRECTION BIAS BY HEAD MOUNTED DISPLAYS

Center point in real space

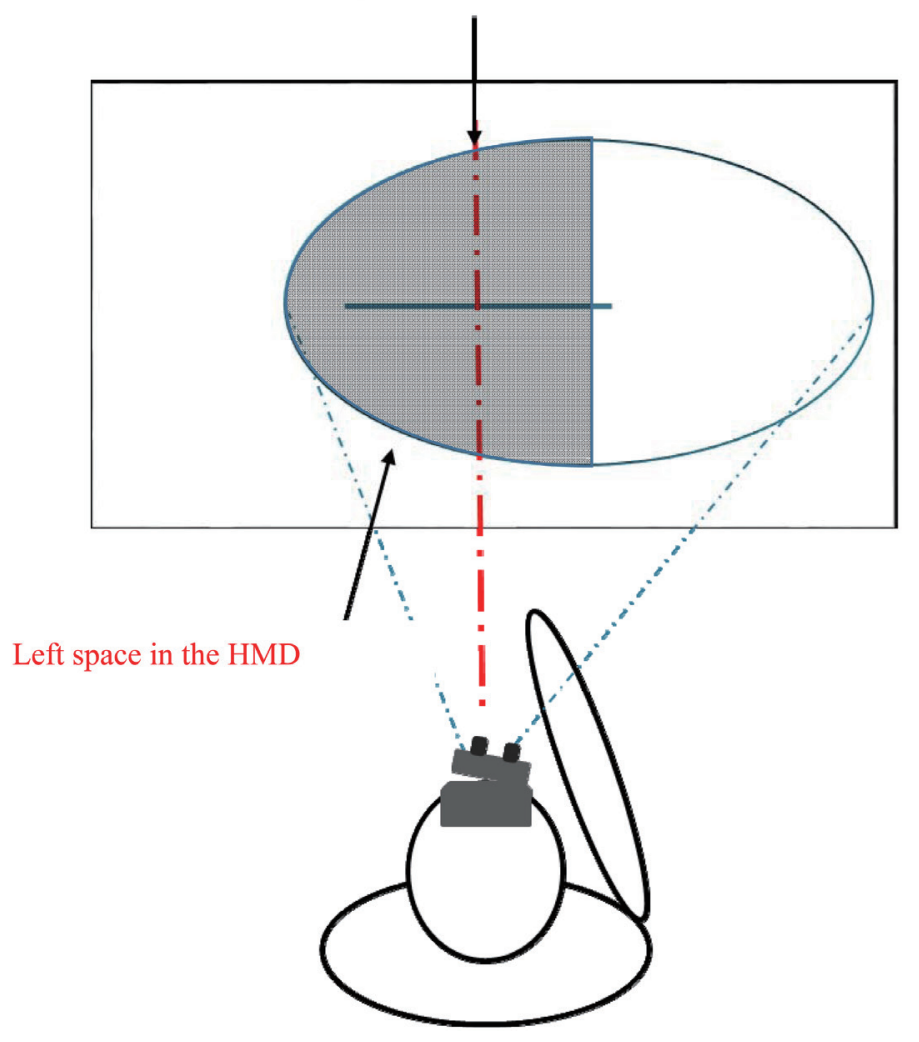

Fig. 4. Relationship between real space and the line segment position in the head-mounted display (HMD). 
structed to move the pointer projected on the monitor to the center point of the line also shown on the monitor. Movement of the mouse pointer did not require hand-eye coordination involving the transfer of proprioceptive information to real space. Because both the line segment and the manipulation of the mouse to move the pointer were visually perceived, it may have been easier to accurately position the mouse on the line segment than it was to position their arm or a stick. The condition in which participants used their right index finger to point to the line segment on the closer monitor elicited the greatest leftward displacement. This finding may have resulted from participants having sufficient experience coordinating hand and eye movements when they used their dominant hand and reached with their own arm and not a stick (i.e., visual motion adaptation) ${ }^{8,9)}$ From this result, it is suggested that there will possibly be a difference in the number of times required until relearning takes place between reaching using the index finger to the proximal space and reaching using the stick to the distal space when performing prism adaptation (PA) ${ }^{10)}$ which is a treatment for USN patients. In other words, it is suggested that it is necessary to change the number of reaching actions in PA in USN with proximal spatial dominance and USN with distal spatial dominance. However, to verify this, it would be necessary to add an adaptation process in this experiment, to increase the number of subjects, and to conduct further research by evenly allocating subjects based on their growth and sports histories. It is also necessary to evaluate the effect of the actual PA intervention with USN patients as subjects.

\section{ACKNOWLEDGMENTS}

We would like to express our appreciation to Mr. Ryusuke Yamada, Manager of the Rehabilitation Department, Niiza Hospital, Japan, and Mr. Hideyuki Tani, Manager of the Rehabilitation Department, Higashiyamato Hospital, Japan, for providing the experimental site and staff.

\section{CONFLICTS OF INTEREST}

There are no conflicts of interest to declare.

\section{REFERENCES}

1. Katz N, Hartman-Maeir A, Ring H, Soroker N: Functional disability and rehabilitation outcome in right hemisphere damaged patients with and without unilateral spatial neglect. Arch Phys Med Rehabil 1999;80:379-384. PMID:10206598, DOI:10.1016/ S0003-9993(99)90273-3

2. Denes G, Semenza C, Stoppa E, Lis A: Unilateral spatial neglect and recovery from hemiplegia: a followup study. Brain 1982;105:543-552. PMID:7104665, DOI:10.1093/brain/105.3.543

3. Cubelli R: Definition: spatial neglect. Cortex 2017;92:320-321. PMID:28454716, DOI:10.1016/j.cortex.2017.03.021

4. Longo MR, Lourenco SF: On the nature of near space: effects of tool use and the transition to far space. Neuropsychologia 2006;44:977-981. PMID:16243365, DOI:10.1016/j.neuropsychologia.2005.09.003

5. McIntosh RD, Ietswaart M, Milner AD: Weight and see: line bisection in neglect reliably measures the allocation of attention, but not the perception of length. Neuropsychologia 2017;106:146-158. PMID:28923304, DOI:10.1016/j.neuropsychologia.2017.09.014

6. Facchin A, Beschin N, Pisano A, Reverberi C: Normative data for distal line bisection and baking tray task. Neurol Sci 2016;37:1531-1536. PMID:27259570, DOI:10.1007/s10072-016-2626-6

7. Jewell G, McCourt ME: Pseudoneglect: a review and meta-analysis of performance factors in line bisection tasks. Neuropsychologia 2000;38:93-110. PMID:10617294, DOI:10.1016/S0028-3932(99)00045-7

8. Iriki A, Tanaka M, Iwamura Y: Coding of modified body schema during tool use by macaque postcentral neurones. Neuroreport 1996;7:2325-2330. PMID:8951846, DOI:10.1097/00001756-199610020-00010

9. Ostry DJ, Gribble PL: Sensory plasticity in human motor learning. Trends Neurosci 2016;39:114-123. PMID:26774345, DOI:10.1016/j.tins.2015.12.006

10. Rossetti Y, Rode G, Pisella L, Farné A, Li L, Boisson $\mathrm{D}$, Perenin MT: Prism adaptation to a rightward optical deviation rehabilitates left hemispatial neglect. Nature 1998;395:166-169. PMID:9744273, DOI:10.1038/25988 